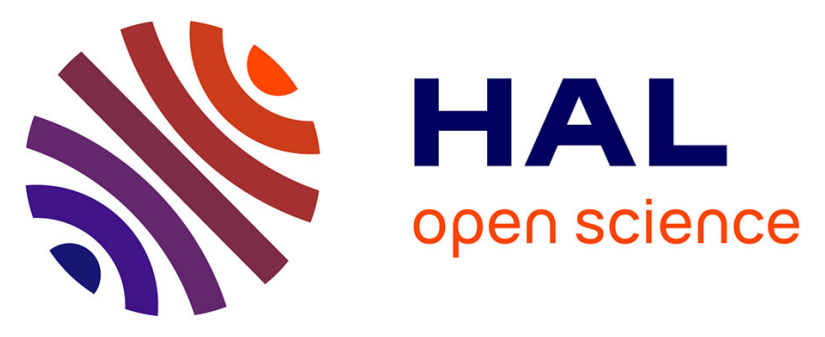

\title{
Development and assessment of a Wake Vortex characterization algorithm based on a hybrid LIDAR signal processing
}

Alexandre Hallermeyer, Agnès Dolfi-Bouteyre, Matthieu Valla, Laurent Le Brusquet, Gilles Fleury, Ludovic Thobois, Jean-Pierre Cariou, Matthieu Duponcheel, Grégoire Winckelmans

\section{To cite this version:}

Alexandre Hallermeyer, Agnès Dolfi-Bouteyre, Matthieu Valla, Laurent Le Brusquet, Gilles Fleury, et al.. Development and assessment of a Wake Vortex characterization algorithm based on a hybrid LIDAR signal processing. 8th AIAA Atmospheric and Space Environments Conference, Jun 2016, Washington, United States. 10.2514/6.2016-3272 . hal-01377098

\section{HAL Id: hal-01377098 \\ https://hal-centralesupelec.archives-ouvertes.fr/hal-01377098}

Submitted on 6 Oct 2016

HAL is a multi-disciplinary open access archive for the deposit and dissemination of scientific research documents, whether they are published or not. The documents may come from teaching and research institutions in France or abroad, or from public or private research centers.
L'archive ouverte pluridisciplinaire HAL, est destinée au dépôt et à la diffusion de documents scientifiques de niveau recherche, publiés ou non, émanant des établissements d'enseignement et de recherche français ou étrangers, des laboratoires publics ou privés. 


\title{
Development and assessment of a Wake Vortex characterization algorithm based on a hybrid LIDAR signal processing
}

\author{
A. Hallermeyer* \\ ONERA - LRS - LEOSPHERE, FRANCE \\ A. Dolfi-Bouteyre ${ }^{\dagger}$ and M. Valla ${ }^{\dagger}$ \\ ONERA, Palaiseau, FRANCE \\ L. Le Brusquet $\ddagger^{\ddagger}$ and G. Fleury ${ }^{\S}$ \\ Laboratoire Signaux et Systèmes (CentraleSupélec, CNRS, Univ Paris-Sud), Gif-sur-Yvette, FRANCE \\ L. Thobois ${ }^{\natural}$ and J.P. Cariou" \\ M. Duponcheel ${ }^{* *}$ and G. Winckelmans ${ }^{\dagger \dagger \dagger}$ \\ LEOSPHERE, Orsay, FRANCE \\ Université Catholique de Louvain, Louvain-la-Neuve, BELGIUM
}

\begin{abstract}
Since air traffic is in constant expansion, a more efficient optimisation of the airports capacity is expected. In this context, the characterization of aircraft hazardous turbulences known as wake vortices with an operational vortex Lidar is one of the major issues for the dynamic distances separation. A study has been probed to develop a hybrid vortex algorithm, i.e that uses both the velocity envelopes and a parametric estimator in the interest of processing time as short as possible. The aim is to make this algorithm exploitable for operational projects. That is why a methodology has been set up to evaluate its precision and its robustness. The results of tests on simulated scenarios of different aircraft vortices and different weather conditions show that this algorithm is able to localize precisely wake vortices and to estimate accurately their circulation in a short time.
\end{abstract}

\section{Nomenclature}

$\Gamma \quad$ Wake Vortex Circulation, $\mathrm{m}^{2} / \mathrm{s}$

$\Gamma_{0} \quad$ Wake Vortex Initial Circulation, $\mathrm{m}^{2} / \mathrm{s}$

$v_{r} \quad$ Radial Velocity, $\mathrm{m} / \mathrm{s}$

$f_{d} \quad$ Doppler Shift, $H z$

$b_{0} \quad$ Initial vortex spacing, $m$

$r_{c} \quad$ Vortex core radius, $m$

\section{Introduction}

In a context of an ever expanding air traffic, the capacity of the international airports with high flows is a major issue in terms of landing and take off throughput. These flows are mostly limited by the presence of pairs of contra-rotating wake vortices created by aircraft on their way through the atmosphere and that are the results of the air flow generating a lift force on a wing profile with a finite span. The characterization

\footnotetext{
*PhD Student, Theoretical and Applied Optics Department, Chemin de la Hunière

†Senior Researcher, Theoretical and Applied Optics Department, Chemin de la Hunière

¥Teacher-researcher, L2S, UMR CNRS 8506, CentraleSupélec-CNRS-Université Paris-Sud, 3 Rue Joliot-Curie

§Supervisor, L2S, UMR CNRS 8506, CentraleSupélec-CNRS-Université Paris-Sud, 3 Rue Joliot-Curie

ฯ Aviation weather research manager, Scientific and Technology Research Department, 16 Avenue Jean Rostand

" Director Scientific and Technology Research Department, 16 Avenue Jean Rostand

** Research Engineer, Institute of Mechanics, Materials and Civil Engineering, Bât Stévin, PLace du Levant 2

${ }^{\dagger \dagger}$ Professor, Institute of Mechanics, Materials and Civil Engineering, Bât Stévin, PLace du Levant 2
} 
of these wake vortices is required for the dynamic distances separation between consecutive aircraft. The measurements of such wake turbulence are made thanks to the reference sensor in this area, the pulsed coherent Doppler LIDAR. The main features of a wake vortex sought are its strength characterized by its circulation $\Gamma$ and its position. In the literature, two notable families of algorithm aiming to retrieve these features from the LIDAR velocity spectra can be found. The first one is a non-parametric method, based on extracting the positive and negative velocity envelopes (VE) using a threshold on the spectra ${ }^{1-3}$ that depends on the SNR and the aircraft category and so needs to be adapted by the user. In the velocity spectra, every velocity value encountered by the laser pulse in the measurement volume is represented. The maximum (and minimum) velocity is the velocity of the wake vortex's field line tangent to the laser beam. The extrema of each velocity spectra gives the wake vortex envelope (positive and negative). These envelopes provide the positions (where the positive envelope has its maximum, and the negative envelope has its minimum). Then to compute the circulation, different methods are exploitable like integrating these envelopes at the range of the vortex core between 5 and 15 meters. In that case the circulation is called $\Gamma_{5-15}$. The second family gathers the algorithms making use of estimators such as the maximum likelihood estimation on the spectra from a Wake Vortex analytical models ${ }^{4}$ or as the maximum of a correlation function between the radial velocities (RV) and a vortex model. ${ }^{5}$ In section 2 of this paper, after a presentation of the models used, a hybrid algorithm is presented. This algorithm is divided into 3 steps based on research of extrema of velocities envelopes and the optimization of a least-squares and a maximum likelihood criteria both based on the Kaden-Winckelmans model. Then in section 3, the results of its performances evaluation on simulated scenarios from both simple simulations and Large Eddy Simulations (LES) are then exposed. In order to evaluate the robustness of the algorithm towards the wake vortex model, a cross check study has been performed using two different models for air motion simulation and for LIDAR vortex parameters estimation algorithm.

\section{Models and Algorithm}

\section{A. Models}

In order to evaluate any algorithm, simulated data have been generated. Indeed, simulation allows to realize various scenarios with realistic and common weather conditions and different configurations of LIDAR optical architecture. With simulations, the initial parameters are under control which means that a ground truth is available and so statistical analysis such as Monte Carlo or sensitivity analysis can be performed. This would not be possible with measurements. Besides, with real data the exact position of the vortices core remains uncertain.

The vortices circulation $\Gamma$ is a quantity proportional to the weight $M$ of the airplane and inversely proportional to its speed $V$ and wingspan $b$, to the air density $\rho$ and the wing loading factor $s$ considered the same for any aircraft of which value $s=\pi / 4$, corresponds to an elliptical loading. Knowing these parameters, it is possible to compute the value of the initial circulation $\Gamma_{0},{ }^{6}$ that is the circulation once the wake roll-up is completed and at its peak strength with:

$$
\Gamma_{0}=\frac{M g}{\rho s b V}
$$

The wake vortex analytical model of Kaden-Winckelmans has been considered to generate the reference data set. This model is a "two-scales" vortex model, with two degrees of freedom ${ }^{7}$ and so is superior in term of azimuthal velocity and circulation profile. ${ }^{8}$

The velocity expression at a point $M$ distant from the center of $r$ for this model considering a wake vortex with a global circulation $\Gamma_{0}$ is defined as follows:

$$
v(r)=\frac{\Gamma_{0}}{2 \pi r} \frac{\alpha\left(\frac{r}{b_{0} / 2}\right)^{1 / 2}}{\left[1+(\alpha-1)\left(\frac{r}{b_{0} / 2}\right)\right]}
$$

where $\alpha$ is an non-dimensional constant rounded at $1.8^{9}$ and $b_{0}$ is the initial vortex separation. This expression of the velocity allows to draw the velocity profile in figure 1 from the center of the vortex to its outer part. Note that this model has the particularity to have no core radius, i.e the maximum radial velocity is reached at its center. This modelling of the core is certainly not realistic but on the other hand 
the description of the vortex velocities beyond is better than other models. Another advantage is that this model is quite simple because once the positions determined, only 2 parameters are to be estimated, the circulations of both vortices which decreases the processing time compared to other models depending on the vortex core radius.

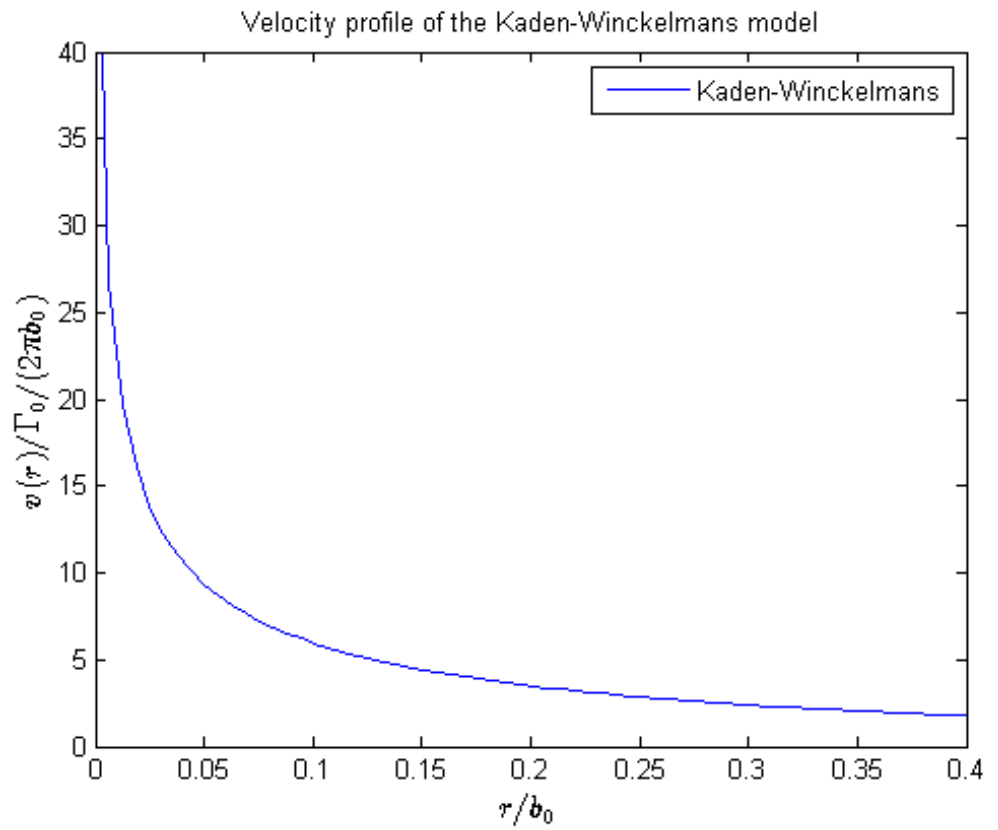

Figure 1. Radial velocity profile of a Kaden-Winckelmans Wake Vortex

Since the LIDAR only measures the radial velocities $v_{r}$, the velocities from equation 2 are projected on the LIDAR axes represented by the Lines of Sight (LOS). Each LOS is associated to the signal proceeding from a single laser pulse and divided into sampling periods called range gates that overlap on one another resulting in a virtual display resolution but nevertheless thinner. Then considering $\lambda$ the laser wavelength and $f_{I F}$ the LIDAR reference frequency, the associated Doppler shifts $f_{d}$ induced by the movement are easily calculated thanks to the following equation:

$$
f_{d}=f_{I F}-\frac{2 v_{r}}{\lambda}
$$

From these Doppler shifts, a simulator of a Pulsed Heterodyne Doppler LIDAR for which signal processing, optical, electronic, mechanic and atmospheric parameters can be freely chosen, is used. It simulates the emission of laser pulses in the atmosphere of which the aerosol particles response is modelized using the feuilleté model. ${ }^{10,11}$ The reference pulsed LIDAR accumulates the periodograms and so does this simulator. Pulsed Fiber LIDAR can have a very high Pulse Repetition Frequency (PRF) that enables large pulse accumulation in order to increase the SNR. ${ }^{12}$

\section{B. Algorithm}

The algorithm evaluated in this paper allows to determine the position of each vortex of a pair and to estimate accurately their circulations. It can be seen as a hybrid algorithm since it uses principles from both families previously presented in introduction. It can be decomposed into three main steps.

The first step is dedicated to the detection and localization of pairs of vortices. The detection is performed thanks to the computation of the moment number two of the spectra, i.e the spectral broadening. The search for wake vortices is made in a pre-delimited zone of the scanning area that corresponds to the window through which the aircraft are supposed to pass. The presence of vortices is determined by a spectral broadening much more elevated than the average. The localization of each vortex is then performed by using 
the velocity envelopes ${ }^{1,13}$ (figure 2). These are obtained for each range gate by using a threshold established from the maximum and the standard deviation of the spectral noise. The indexes of the range gates corresponding to the position of both vortices are determined by the position of highest difference between maximum of the positive envelope (Vmax) and the minimum of the negative envelope (Vmin) (see figure 3). Next, considering these two range gates, in order to get the associated angles for each vortex, the median index of line of sight between the index of the maximum of the positive envelope and minimum the minimum of the negative envelope is taken. The localization can be biased, especially in a low aerosol concentration or high turbulence situations. This is why that first step is mainly used to get a first approximate localization of the vortices. It allows then to narrow the area of interest so that the processing time for the following functions is decreased.

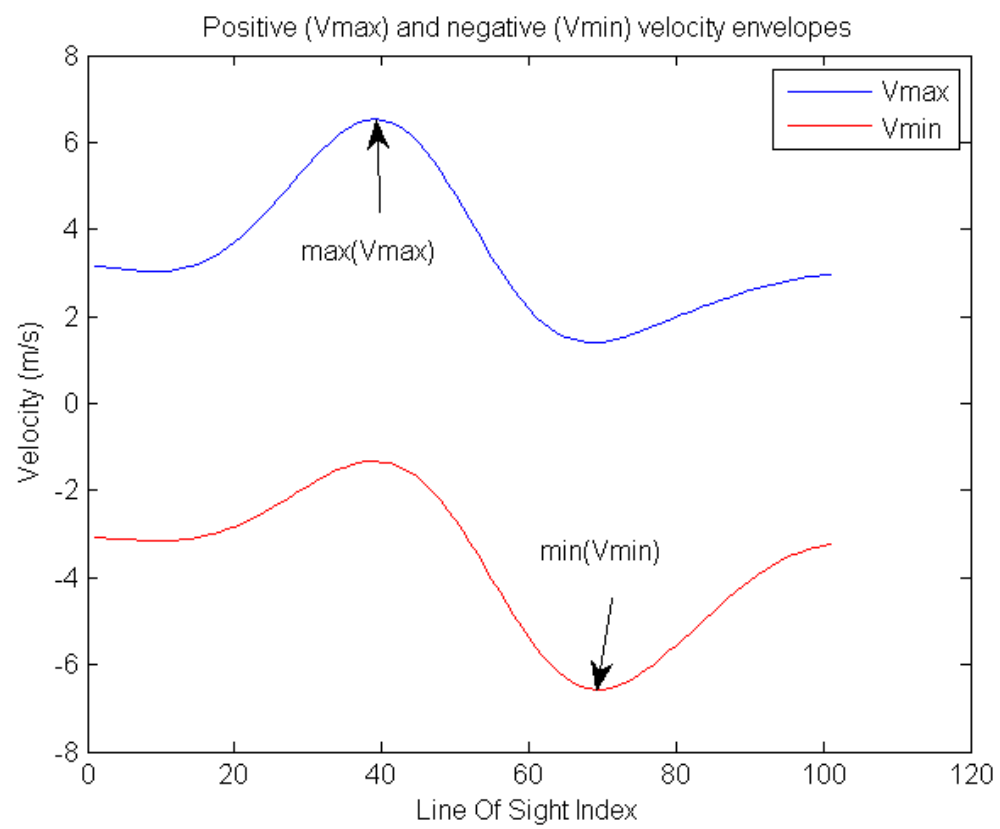

Figure 2. Representation of the positive and negative velocity envelopes for the range gate 81 (case of a nearest vortex)

The second step acts as a refiner for the vortices positions given by step 1 and also acts as a first estimator of the circulations. Indeed, the circulations are directly conditioning the spectra shapes and so must be left as free parameters in this estimation step. It is based on minimizing the criterion of the least-squares:

$$
L S(\Theta)=\sum_{m=1}^{M} \sum_{n=1}^{N}\left\|Y_{m, n}-S_{m, n}(\Theta)\right\|^{2}
$$

$Y_{m, n}$ is the observed periodogram of the $n$-th of the $N$ range gates of the $m$-th of the $M$ lines of sight and $S_{m, n}(\theta)$ is the spectra simulated with the vortex parameters vector $\Theta$ for the same range gate. Here $\Theta$ is composed by the two circulations and the horizontal and vertical positions for both vortices. For the positions, it is initialized with the ones output by step 1 and for the circulations a starting point is arbitrarily taken at $300 \mathrm{~m} / \mathrm{s}$ and $-300 \mathrm{~m} / \mathrm{s}$. This criterion is a non-linear least-squares optimized for algorithms like the Levenberg-Maquardt algorithm.

The final step is a maximum likelihood based estimation partially inspired by the theoretical study led by Frehlich ${ }^{4}$ but instead of estimating 6 parameters (circulation, height and range for both vortices), it concentrates on estimating the circulations of the vortices only. The positions are used as fixed parameters because, first, they have already been accurately determined and second, it makes the estimator more efficient, meaning that it is more stable and it greatly decreases the computation time needed to maximize the log-likelihood. The initial spacing $b_{0}$ is then given by the distance between the two centers. As the 


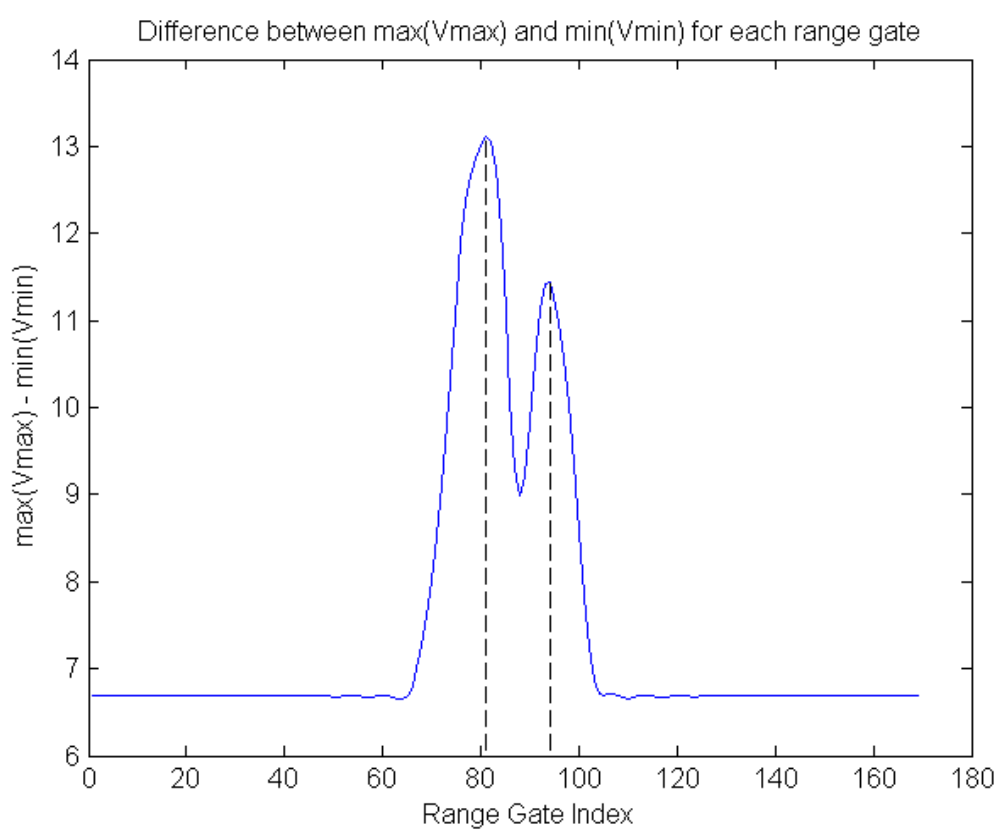

Figure 3. Representation of the difference between the maximum of the positive velocity envelope and the minimum of the negative velocity envelope for each range gate. The two maxima determine which range gates are the most centered on the vortices

vortex models cannot generally represent well the velocity near the cores (it is particularly true for the Kaden-Winckelmans model), the used criterion is balanced so as to ignore the part of the data where the models are poorly specified, i.e the region between $r=0$ and $r=0.1 b_{0}$. This choice leads to gains with respect to the robustness. The expression of the log-likelihood of the $n$-th range gate and of the $m$-th line of sight used, $L L_{m, n}$ is:

$$
L L_{m, n}(\theta)=c s t-A \sum_{k=1}^{K}\left[\ln S_{m, n}(k, \theta)+\frac{Y_{m, n}(k)}{S_{m, n}(k, \theta)}\right]
$$

In the feuilleté model, the atmosphere is sliced into sections of aerosol particles. The signals backscattered by theses slices are considered as white Gaussian centered noises uncorrelated from one slice to another. Considering a single range gate which is the aggregation of several aerosols sections, as a sum of independent Gaussian variable, its signal is then a white Gaussian centered noise and so is its associated Fourier Transform. Finally, by taking the periodogram of $A$ accumulated spectra, a $\chi^{2}$ distribution at $2 A$ degrees of freedom within a constant depending from $\theta$ is obtained, due to the manipulation of Fourier Transform in complex ${ }^{4,14}$ and because there is no zero-padding done. $\theta$ is the vortex parameters vector composed by only the two circulations. The initial point for the maximization is the estimates of the circulations output by step 2. $k$ is the spectral index, $K$ the total number of spectral bins for one spectra and $A$ is the number of accumulated pulses, cst being a constant. The global likelihood that is to be maximized, is the sum over $n$ and $m$ of the $L L_{m, n}$.

\section{Experiments and Results}

In this section, the goal is to study the influence on the algorithm performances of the aerosol density in the atmosphere. The root-mean-square error (RMSE) in equation 6 is used as performances evaluation parameter.

$$
\operatorname{RMSE}(\hat{\theta})=\sqrt{\operatorname{Var}(\hat{\theta})+(\operatorname{Bias}(\hat{\theta}, \theta))^{2}}
$$




\section{A. Simulations}

Aiming to acquire a data base that describes most of the airports traffic as well as diverse weather conditions, it is compulsory to consider different scenarios of Signal to Noise Ratio associated to the aerosols backscattering coefficient, of aircraft category and of atmospheric turbulence. With this in mind, simulations are realized for:

- The three most wide-spread categories of aircraft: Medium, Heavy and Jumbo.

- High, standard and low SNR (proportional to the density of aerosol particles that is characterized by the back-scattering coefficient)

- Presence of EDR (Eddy Dissipation Rate): The EDR is associated to the ambient turbulence that plays a major role in the vortices circulation decay. Indeed, the interaction between the outer part of a vortex and the atmosphere accelerates the vortex energy dissipation. In these simulations, the hypothesis of an additive velocity field to the crosswind and to the vortices wind field is made. This hypothesis is only valid in presence of weak turbulence, that is why the simulations limit themselves to a low EDR case.

For each category of aircraft, one hundred realizations of each atmospheric scenario are made in a $5 \mathrm{~m} / \mathrm{s}$ crosswind, the turbulence and the LIDAR noise being randomly generated. The LIDAR is simulated as if it was positioned perpendicularly to the runway at a distance of $500 \mathrm{~m}$ and scanning from twelve to zero degrees and reaching a distance of $1 \mathrm{~km}$. The position of the LIDAR to the vortices position is illustrated in figure 4. For the rest of the study, the nearest vortex will be considered as the left vortex and the farthest as the right vortex.

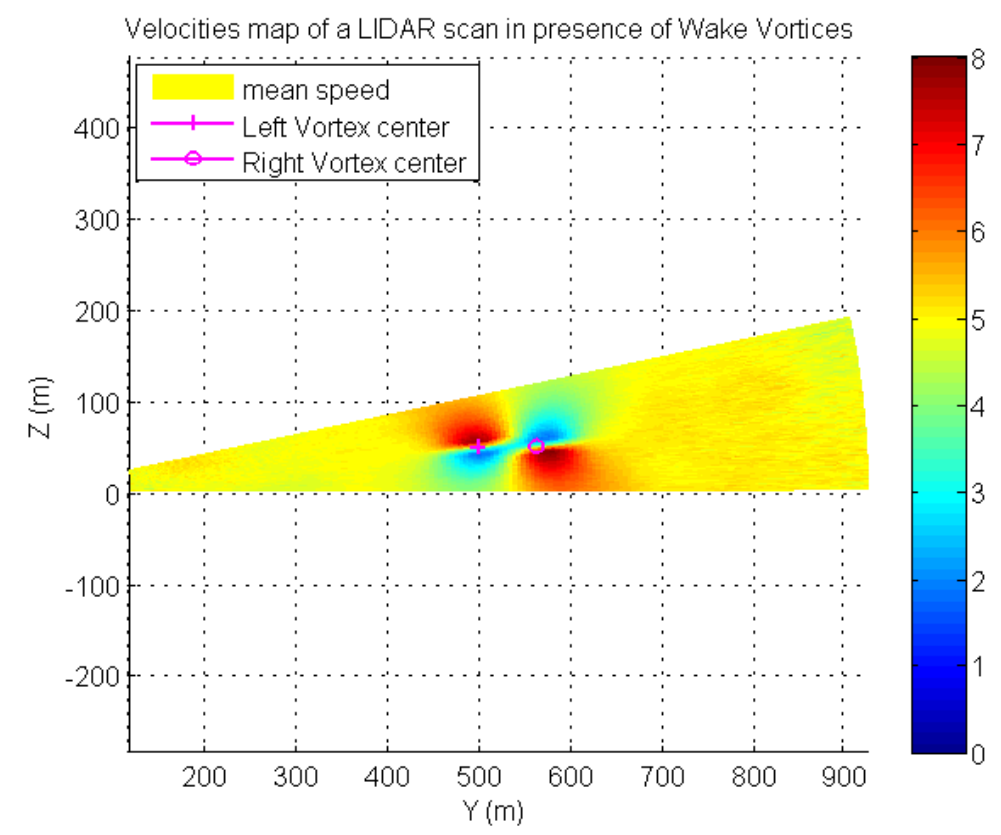

Figure 4. Example of LIDAR scan with Wake vortices

The initial vortex circulation and spacing used for each category of aircraft are gathered in table 1 .

For this study, the LIDAR simulator is set with the configuration of the Windcube 200s in vortex mode. The Gaussian pulses are emitted at a repetition frequency of $20 \mathrm{kHz}$ and have a Full Width at Half Maximum (FWHM) of $200 \mathrm{~ns}$. The sampling corresponding to a range gate is made during $256 \mathrm{~ns}$ at a rate of $250 \mathrm{MHz}$. The offset that gives a thinner display resolution in distance although virtual, between two consecutive range gates is $32 \mathrm{~ns}$. In this configuration, the accumulation that provides a higher SNR is made during $50 \mathrm{~ms}$. 


\begin{tabular}{ccc}
\hline Aircraft type & Circulation $\Gamma_{0}\left(\mathrm{~m}^{2} / \mathrm{s}\right)$ & Initial spacing $b_{0}(\mathrm{~m})$ \\
\hline Medium & 250 & 26.7 \\
Heavy & 430 & 47.7 \\
Jumbo & 600 & 62.6 \\
\hline
\end{tabular}

Table 1. Initial vortex circulation and spacing

The overall efficiency of the localization process of the vortices is illustrated here by the aircraft category with the smallest vortex spectral signature so theoretically the most delicate to locate precisely, the medium class. The results of the estimations at low EDR are gathered in table 2. For each component of the vortex location, height $(\mathrm{Z})$ and horizontal position $(\mathrm{Y})$, and for each back-scattering value, the mean bias (left) and the standard deviation (right) are presented. Figure 5 represents the scattering of the estimates of the position for the left vortex.

\begin{tabular}{|c|c|c|c|c|c|c|c|}
\hline \multicolumn{2}{|c|}{ Back-scattering coefficient } & \multicolumn{2}{|c|}{$10^{-8} \mathrm{~m}^{-1} / \mathrm{sr}$} & \multicolumn{2}{|c|}{$10^{-7} \mathrm{~m}^{-1} / \mathrm{sr}$} & \multicolumn{2}{|c|}{$10^{-6} \mathrm{~m}^{-1} / \mathrm{sr}$} \\
\hline \multirow{3}{*}{ Left Vortex } & $\mathrm{Y}$ & $-0.94 \mathrm{~m}$ & $1.71 \mathrm{~m}$ & $-0.85 \mathrm{~m}$ & $1.34 \mathrm{~m}$ & $-1.47 \mathrm{~m}$ & $1.35 \mathrm{~m}$ \\
\cline { 2 - 8 } & $\mathrm{Z}$ & $-0.26 \mathrm{~m}$ & $0.38 \mathrm{~m}$ & $-0.09 \mathrm{~m}$ & $0.21 \mathrm{~m}$ & $-0.14 \mathrm{~m}$ & $0.19 \mathrm{~m}$ \\
\hline \multirow{2}{*}{ Right Vortex } & $\mathrm{Y}$ & $1.68 \mathrm{~m}$ & $1.83 \mathrm{~m}$ & $0.59 \mathrm{~m}$ & $0.97 \mathrm{~m}$ & $-0.74 \mathrm{~m}$ & $1.49 \mathrm{~m}$ \\
\cline { 2 - 8 } & $\mathrm{Z}$ & $0.26 \mathrm{~m}$ & $0.33 \mathrm{~m}$ & $0.07 \mathrm{~m}$ & $0.16 \mathrm{~m}$ & $-0.05 \mathrm{~m}$ & $0.19 \mathrm{~m}$ \\
\hline
\end{tabular}

Table 2. Mean biases (left subcolumns) and standard deviations (right subcolumns) of the vortices localization estimates for the Medium class

The localization is little affected by the Signal to Noise Ratio although the worst performances are obtained when the aerosol particles density is very low, i.e when the SNR is low. However, given the LIDAR display resolution of $5 \mathrm{~m}$ and the angular resolution at such a distance that is about $1.5 \mathrm{~m}$, the localization steps are quite accurate since the results are below these resolutions.

Since the results are quite similar, only the results for the left vortex will be presented. Figure 6 gathers the root-mean-square errors and the standard deviations of the estimates of circulations for all categories and back-scattering coefficient after the least-squares step and after the maximum likelihood estimation step. By comparing these two histograms, it is clear that the last step making use of the likelihood improves the circulation estimation accuracy but for the heavy case in low SNR and only because the result after the step 2 is very good. Indeed, in every other case the mean-square error is decreased after step 3 , largely by diminishing the bias, the standard deviation being already quite low after step 2. When comparing the results for a single aircraft category, as expected, the RMSE decreases when the back-scattering coefficient increases, i.e the SNR increases. The maximum likelihood estimation can therefore be qualified as a circulation refiner.

A study that will highlight the time saved by using this hybrid algorithm instead of estimating directly 6 parameters thanks to the likelihood is currently in progress. The results will be exhibited in the final version of this paper.

\section{B. Influence of the analytical model}

In this section, the results of a cross-model study is presented. A data set in the same conditions than exposed previously but using a Hallock-Burnham model is generated. The radial velocity of a point $M$, distant from the center of a Hallock-Burnham wake vortex of $r$ is given by:

$$
v(r)=\frac{\Gamma_{0}}{2 \pi} \frac{r}{r^{2}+r_{c}^{2}}
$$

By comparison with the Kaden-Winckelmans, this model has a core radius that is proportional to the 


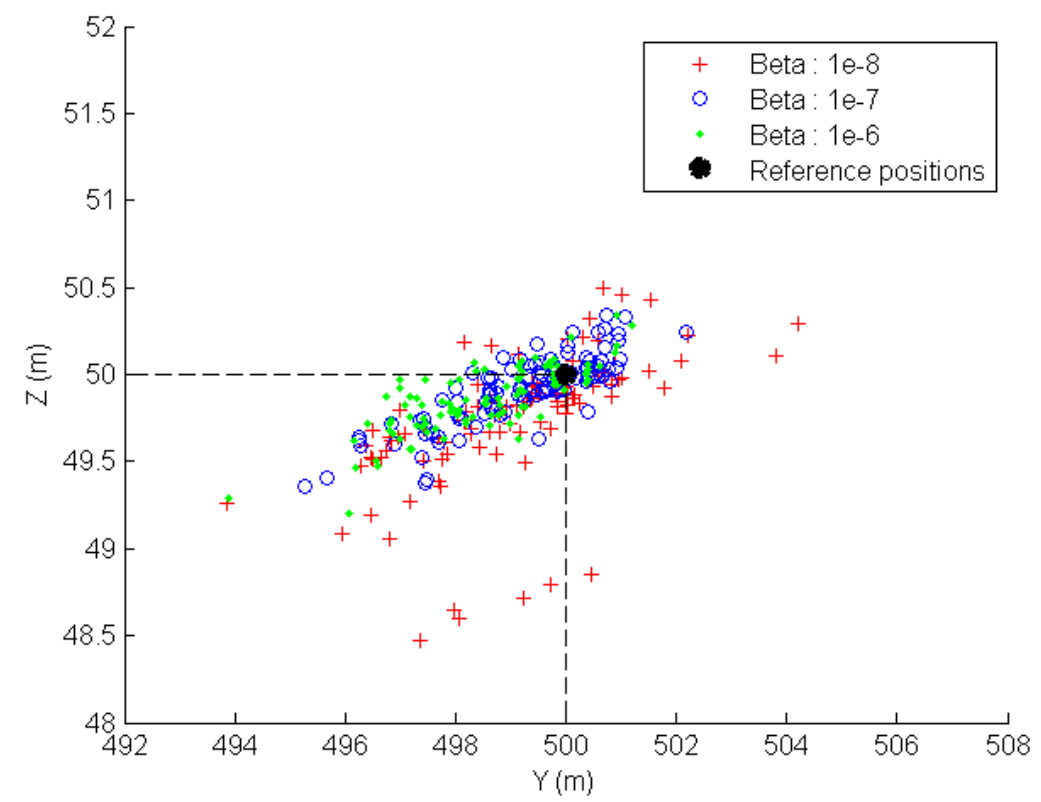

Figure 5. Estimated positions of the left vortex (Medium class case) after the refining step by minimizing the criterion of the Least-Squares
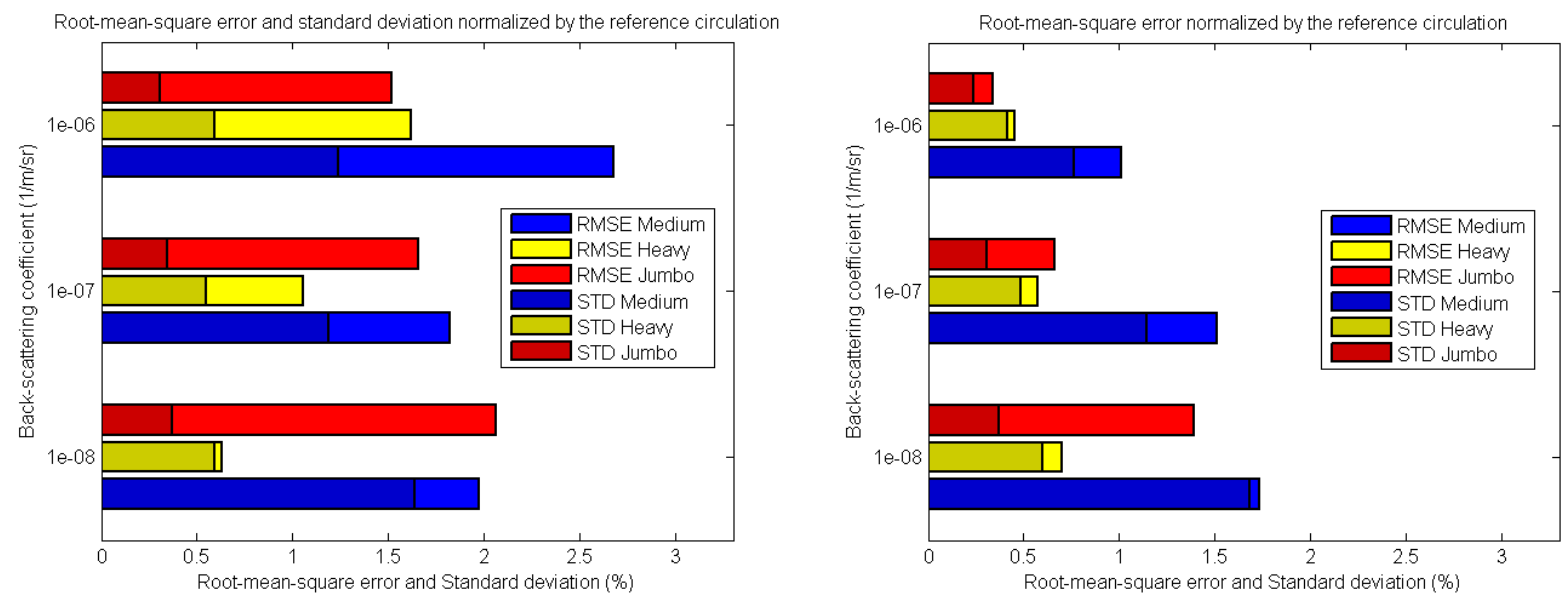

Figure 6. Root-mean-square error and standard deviation (darker) of the estimated circulations for all aircraft categories output after the least squares minimization and after the log-likelihood maximization. The reference circulations being different according to each category of aircraft, the results are presented normalized by theses circulations to facilitate the comparison cross-categories

initial vortex spacing : $r_{c}=0.05 b_{0}$.

Figure 7 represents the velocity profile for both the Kaden-Winckelmans and the Hallock-Burnham models for the same value of circulation. For the Hallock-Burnham model, the maximum velocity $v_{\max }$ is definitely reached for $r=r_{c}$.

In order to study the differences generated by the use of different analytical models to realize the wake vortex scenarios and to make the estimation, the positions of the wake vortices are supposed perfectly known. Only the step 3 of the algorithm is then used with a starting point taken at $300 \mathrm{~m} / \mathrm{s}$ and $-300 \mathrm{~m} / \mathrm{s}$. The 


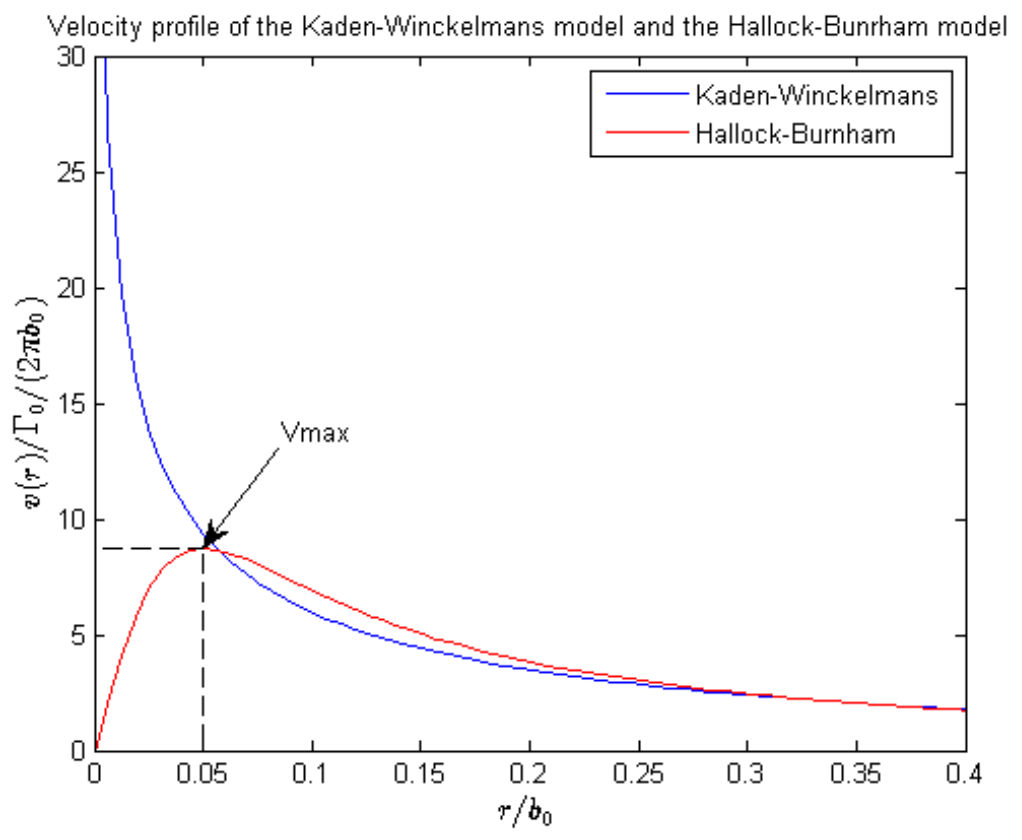

Figure 7. Radial velocity profiles for the Hallock-Burnham and Kaden-Winckelmans models

root-mean-square errors and the standard deviations of the circulations estimations obtained for this crossmodel study are presented in figure 8 .

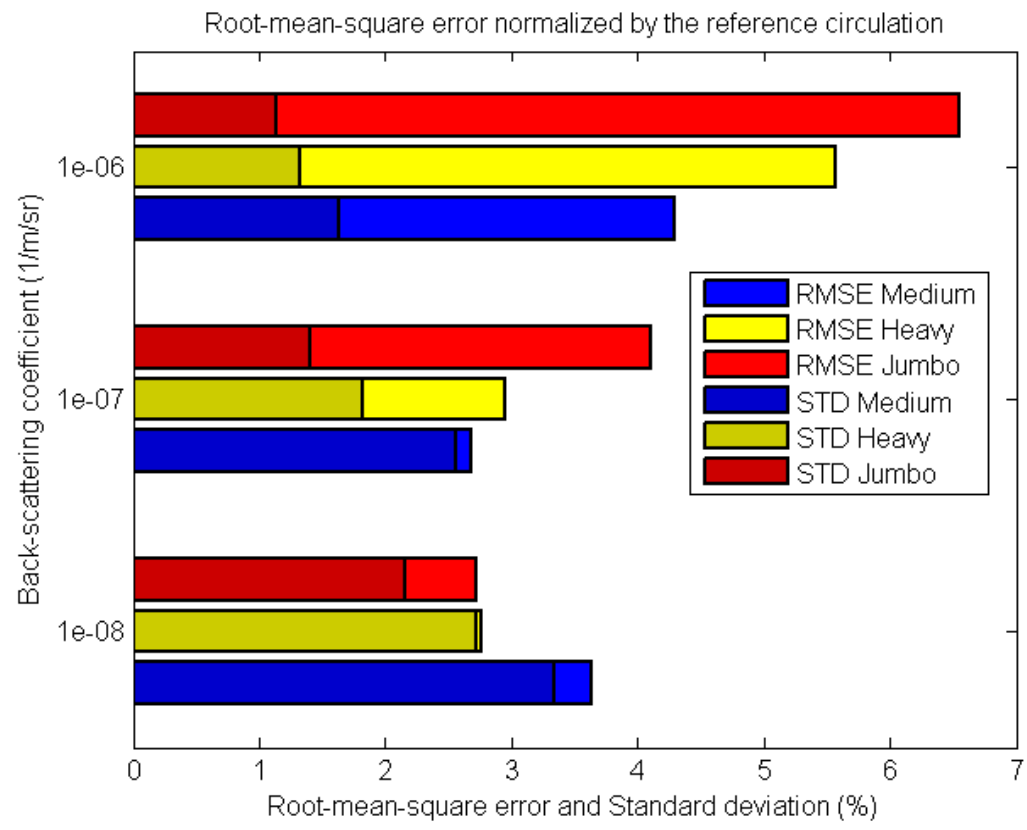

Figure 8. Root-mean-square error and standard deviation (grey) of the estimates of Hallock-Burnham wake vortices scenarios by maximizing the log-likelihood based on the Kaden-Winckelmans model for all aircraft categories.

It can be seen that for every category the standard deviation decreases when the back-scattering coef- 
ficient increases which proves that the algorithm still behaves correctly towards the Signal to Noise Ratio. However, the error greatly increases with the SNR which conveys the differences between the two models via the bias. Indeed, these differences at the level of the spectral signature are heightened with the increasing SNR.

The accumulated spectra can be seen as histograms of the velocities. The correlation depth, meaning the area tangential to the field line where the aerosols have an equivalent radial velocity, is bigger while moving away from the center of the vortex. When measuring too close to the center, between 0 and $0.05 b_{0}$ this area is too small compared to the measurement gate to observe any contribution. On the other hand, when measuring too far from the core radius, the velocities linked to the vortex are too weak and are merged with the crosswind. The area with a correlation depth big enough and where velocities linked to the vortex are strong enough is precisely where the two models diverge, between $0.05 b_{0}$ and $0.3 b_{0}$ as represented on figure 7 . At equal circulations the integral of the velocity profile of the Kaden-Winckelmans model is lower than the one of the Hallock-Burnham model between these two bounds. So by using the Kaden-Winckelmans model to estimate the circulation of Hallock-Burnham vortices, it is logical to get greater values of circulations than the reference.

\section{Large Eddy Simulations}

The last part of the validation of this algorithm is based on the LES data provided by UCL (Université Catholique de Louvain). ${ }^{15}$ These data simulate a pair of wake vortices in ground effect that would have been created by the passage of an heavy aircraft such as the A340. The model of Hallock-Burnham was used to generated them and the same LIDAR simulator presented previously allowed to retrieve the spectra. The exploitation of these data is in progress and results will be presented in the final paper.

\section{Conclusion}

The proposed algorithm may be qualified of hybrid since it uses methods from both families of algorithms, the velocity envelopes to localize and then two estimators (least-squares and log-likelihood) to refine the positions and to compute accurately the circulation of wake vortices, has been developed. The main advantage of this three steps strategy is to reach a process as fast as possible. This uncommon algorithm has been evaluated on simulated data that involve different scenarios of wake vortices and weather conditions. The results show that the localization accuracy after the refining step is thinner than the resolutions of the LIDAR (display and angular) whatever the Signal to Noise Ratio. After the final step using the maximum likelihood estimation, the root mean square error is in any case inferior to $2 \%$. Tests are made at the moment, to compare the processing time between this algorithm and a 6 parameters maximum likelihood estimation. A priori, this hybrid processing is faster. When confronting two different analytical models like Kaden-Winckelmans and Hallock-Burnham, it is seen that the decisive area for the estimation of the circulation by the LIDAR is between $0.05 b_{0}$ and $0.3 b_{0}$. A divergence of the velocity profiles is the cause for an increasing bias when the SNR increases. These facts will be very useful for the study of the LES that is currently being led.

\section{References}

\footnotetext{
${ }^{1}$ Köpp, F., Rahm, S., and Smalikho, I., "Characterization of Aircraft Wake Vortices by 2- $\mu$ m Pulsed Doppler Lidar," Journal of Atmospheric and Oceanic Technology, Vol. 21, No. 2, 2004, pp. 194-206.

${ }^{2}$ Wassaf, H. S., Burnham, D., and Wang, F., "Wake Vortex Tangential Velocity Adaptive Spectral (TVAS) Algorithm for Pulsed Lidar Systems," in "16th CLRC meeting, Long Beach, California," , 2011.

${ }^{3}$ Banakh, V. and Smalikho, I., Coherent Doppler wind lidars in a turbulent atmosphere, Artech House, 2013.

${ }^{4}$ Frehlich, R. and Sharman, R., "Maximum likelihood estimates of vortex parameters from simulated coherent Doppler lidar data," Journal of Atmospheric and Oceanic Technology, Vol. 22, No. 2, 2005, pp. 117-130.

${ }^{5}$ Smalikho, I., Banakh, V., Holzäpfel, F., and Rahm, S., "Method of radial velocities for the estimation of aircraft wake vortex parameters from data measured by coherent Doppler lidar," Optics Express, Vol. 23, No. 19, 2015, pp. A1194-A1207.

${ }^{6}$ Gerz, T., Holzaepfel, F., Darracq, D., de Bruin, A., Elsenaar, A., Speijker, L., Harris, M., Vaughan, M., and Woodfield, A., "Aircraft wake vortices: a position paper," Wakenet position paper.

${ }^{7}$ De Visscher, I., Winckelmans, G., Lonfils, T., Bricteux, L., Duponcheel, M., Bourgeois, N., et al., "The WAKE4D simulation platform for predicting aircraft wake vortex transport and decay: Description and examples of application," AIAA Paper, Vol. 7994, 2010, p. 2010.
} 
${ }^{8}$ Winckelmans, G., "Aircraft wake vortices: physics ans UCL models," , 2011. WakeNet3-Europse Specific Workshop "Re-categorization" slides available on www.wakenet.eu.

${ }^{9}$ Winckelmans, G. and De Visscher, I., "RMC-based severity metrics: possibilities and scalings," , 2014. WakeNet-Europe slides available on www.wakenet.eu.

${ }^{10}$ Salamitou, P., Dabas, A., and Flamant, P. H., "Simulation in the time domain for heterodyne coherent laser radar," Applied optics, Vol. 34, No. 3, 1995, pp. 499-506.

${ }^{11}$ Frehlich, R. G. and Kavaya, M. J., "Coherent laser radar performance for general atmospheric refractive turbulence," Applied Optics, Vol. 30, No. 36, 1991, pp. 5325-5352.

${ }^{12}$ Dolfi-Bouteyre, A., Canat, G., Valla, M., Augere, B., Besson, C., Goular, D., Lombard, L., Cariou, J.-P., Durecu, A., Fleury, D., et al., "Pulsed 1.5-m LIDAR for Axial Aircraft Wake Vortex Detection Based on High-Brightness Large-Core Fiber Amplifier," Selected Topics in Quantum Electronics, IEEE Journal of, Vol. 15, No. 2, 2009, pp. 441-450.

${ }^{13}$ Ramsey, D. J. and Nguyen, D. C., "Characterizing Aircraft Wake Vortices with Ground-Based Pulsed Coherent Lidar: Effects of Vortex Circulation Strength and Lidar Signal-to-Noise Ratio on the Spectral Signature," in "3rd AIAA Atmospheric Space Environments Conference,", 2011, p. 3198.

${ }^{14}$ Rye, B. J. and Hardesty, R., "Discrete spectral peak estimation in incoherent backscatter heterodyne lidar. I. Spectral accumulation and the Cramer-Rao lower bound," Geoscience and Remote Sensing, IEEE Transactions on, Vol. 31, No. 1, 1993, pp. $16-27$.

${ }^{15}$ Bricteux, L., Duponcheel, M., De Vissher, I., and Winckelmans, G., "LES investigation of the transport and decay of wake vortices in ground effects and under various crosswind conditions," , 2015. Submitted to Physics of Fluids. 